\title{
The effects of plane rotation on the recognition of brief masked pictures of familiar objects
}

\author{
REBECCA LAWSON \\ University of Liverpool, Liverpool, England \\ and \\ PIERRE JOLICOEUR \\ University of Waterloo, Waterloo, Ontario, Canada
}

\begin{abstract}
Two experiments examined the effects of plane rotation on the recognition of briefly displayed pictures of familiar objects, using a picture-word verification task. Mirroring the results of earlier picture naming studies (Jolicoeur, 1985; Jolicoeur \& Milliken, 1989), plane rotation away from a canonical upright orientation reduced the efficiency of recognition, although in contrast to the results from picture naming studies, the rotation effects were not reduced with experience with the stimuli. However, the rotation effects were influenced by the visual similarity of the distractor objects to the picture of the object presented, with greater orientation sensitivity being observed when visually similar distractors were presented. We suggest that subjects use orientation-sensitive representations to recognize objects in both the present unspeeded verification and in the earlier speeded naming tests of picture identification.
\end{abstract}

It has been reported consistently that plane-disoriented views of familiar objects are named more slowly than upright views of objects (e.g., De Caro \& Reeves, 1995; Jolicoeur, 1985; Jolicoeur \& Milliken, 1989; McMullen \& Jolicoeur, 1990, 1992). In general, a monotonic increase in naming latencies from $0^{\circ}$ to $150^{\circ}$ on initial naming has been observed. The effects of plane rotation reduce with repeated presentation of the same objects (Jolicoeur, 1985), with most of this reduction occurring from the first to the second presentation of a given object. However, plane rotation effects do not decrease if different sets of objects are presented across blocks, or if only upright views of objects are presented across blocks (Jolicoeur \& Milliken, 1989), or if subjects are repeatedly presented with the same set of disoriented objects but are not required to identify them (Murray, 1995).

Jolicoeur (1990) suggested that on initial presentation, subjects may have to transform disoriented stimuli before they can be matched to an upright stored representation. The image transformation is assumed to require more time to normalize increasingly disoriented stimuli. One example of such a transformation process is mental rotation. However, subjects may also extract orientationinvariant features from an image of an object. Under certain circumstances, such features may mediate efficient orientation-insensitive object recognition. For instance,

We wish to thank Michael Corballis, Geoffrey Loftus, and Joan Gay Snodgrass for their helpful comments on an earlier version of this manuscript. This research was supported by Grant LT-558/94 from the International Human Frontier Science Program to R.L. Correspondence should be addressed to R. Lawson, Department of Psychology, University of Liverpool, Eleanor Rathbone Building, Bedford Street South, Liverpool L69 7ZA, U.K. (e-mail: rlawson@liverpool.ac.uk) if subjects are repeatedly required to identify the same set of objects, they may learn to recognize a set of unique, orientation-invariant distinguishing features that allow them to discriminate between the objects in the set. This alternate route to recognition may account for the observed reduction in the effects of plane rotation after repeated presentations of familiar objects (Jolicoeur, 1985; Jolicoeur \& Milliken, 1989; Murray, Jolicoeur, McMullen, \& Ingleton, 1993). Jolicoeur (1990) has termed this account the dual route account, since it proposes that there are two independent routes to recognition: a transform-then-match route and an invariant-features route. A stimulus may be identified by being transformed to a canonical (upright) view and then matched to an orientation-sensitive representation (the transform-then-match route), or orientationinvariant features may be extracted from the stimulus and these features may be matched directly to stored distinguishing object features (the invariant-features route).

However, it has been noted that effects of plane rotation on the identification of familiar objects do not necessarily indicate that subjects recognize disoriented views of objects less efficiently than upright views. Corballis (1988) and De Caro and Reeves (1995) have suggested that subjects may recognize disoriented views just as efficiently as upright views. In order to account for the observed effects of plane rotation on picture naming, they have suggested that the increased naming latencies for disoriented views may be due to subjects' double-checking the identity of an object for these views (perhaps by mentally rotating the stimuli) prior to responding. We will term this account of the effects of plane rotation on object recognition the double-checking account. A strong version of this account would assume that object recognition is mediated by orientation-invariant representations (which encode 
no orientation-specific information), and that all the orientation effects observed in tasks such as the speeded naming of disoriented views of familiar objects are simply due to subjects' double-checking prior to responding.

Corballis (1988) and De Caro and Reeves (1995) have criticized transform-then-match accounts of plane rotation effects by noting that such accounts appear to assume that a subject knows the orientation of an object prior to being able to identify that object. They have argued that if this were not the case, subjects would not know in what way and how far to transform a stimulus in order to normalize it, so that it could be matched to a stored representation. Indeed, a stimulus cannot be known to be disoriented unless it has been identified-a novel, unfamiliar object has no canonical upright orientation. Instead, De Caro and Reeves have claimed that subjects can generally identify an object before they know what the orientation of the object is, and further, that identity information may be used to inform image transformation processes. They have suggested that image transformation processes may be employed to ascertain the orientation and not the identity of disoriented objects.

However, it is not necessarily a paradox for subjects to be able to identify the orientation of an object prior to being able to identify the object. Certain image cues may allow subjects to determine the probable orientation of an object without already knowing what that object is (see, e.g., Ullman, 1989). Physical cues such as the main axis of elongation of the image provide strong evidence as to the orientation of the object (Marr, 1982). Given the effects of gravity, objects are generally at a stable orientation only if their main axis of elongation is perpendicular (e.g., sofas) or parallel (e.g., chairs) to the force of gravity, and objects will be more stable if their base is wider than their top. In addition, axes of symmetry may be informative as to object orientation. Objects are generally symmetrical about axes that are perpendicular and/or parallel to the force of gravity. Finally, features and parts common to a class of objects may give cues about an object's orientation without providing sufficient disambiguating information to identify an object. For example, feet are found at the base of many animals, and wheels are found at the base of many vehicles, but further individuating information is required to identify the particular animal or vehicle depicted.

The current evidence from picture naming studies does not decisively favor either of the two competing accounts of plane rotation effects on the identification of familiar objects-namely, the dual route account and the doublechecking account. To provide converging evidence for or against these two accounts, we used a different task - the identification of briefly presented, masked pictures of objects shown at different plane rotations. In this task, subjects saw a picture of a familiar object presented for $42 \mathrm{msec}$ or less and followed immediately by a pattern mask. Subjects then selected the name of the object that they believed had been presented from three written alternatives. In the next section, we outline the basis by which results from this procedure can help us to discriminate between the two theoretical accounts of plane rotation effects on object identification.

\section{EXPERIMENT 1}

In Experiment 1, familiar objects were presented at one of a range of plane rotations from $0^{\circ}$ (upright) to $180^{\circ}$ (inverted) for either 28 or $42 \mathrm{msec}$ and were then immediately pattern masked. Subjects were instructed to select the name of the object that had been presented from three written alternatives that appeared after the mask. Subjects' responses were unspeeded, so if subjects did perform double-checks prior to responding, this could not have affected their performance. Thus if the increase in naming latencies for disoriented views is due only to subjects' double-checking before responding, then in the unspeeded masking task employed here, no increase in the difficulty of verifying disoriented views relative to upright views would be predicted. Conversely, if the increase in naming latencies for disoriented views results from subjects' transforming disoriented views to a canonical orientation, as Jolicoeur (1990) has proposed in the dual route account, then in the masking task employed here, verification difficulty would be predicted to be affected by plane rotation because disoriented views would not match the stored representation as well as an upright view.

On half the trials in Experiment 1, the two written distractor alternatives named objects that were visually similar to the picture of the object that had been presented (see Appendix). For example, if a picture of a deer was presented, GOAT and DONKEY were the visually similar written alternatives. On the remaining trials, the written distractor alternatives named objects that were visually dissimilar to the object. If a picture of deer was presented, the visually dissimilar distractors were BOWL and BOW.

On the dual route account, we predicted that if subjects were able to use orientation-invariant features to identify an object, such a strategy should be most effective when visually dissimilar distractors were presented. If the depicted object and the distractors are all visually similar, it should be more difficult to find a simple orientation-invariant feature that uniquely identifies the depicted object. In contrast, relatively coarse identifying features such as aspect ratio, texture, or the presence of straight lines or curves will often serve to distinguish reliably among a set of three visually dissimilar objects. For instance, if a picture of a deer was followed by BOWL, DEER, and BOW as the written response alternatives, the deer could be identified reasonably confidently simply by glimpsing its legs or one of many other possible features. However, if similar distractors were presented, there would be fewer suitable features available to distinguish between the depicted object and the distractor items. For example, if a picture of a deer was followed by GOAT, DEER, and DONKEY as the response alternatives, then simply detecting the presence of legs or a head or tail would 
not serve to identify the deer. Only a small number of features (which would often be complex), such as a particular shape of head or the presence of antlers, would be sufficient to discriminate accurately between the alternatives. It would be difficult to reliably extract one of these features from a briefly presented, faint picture. Instead, subjects would be forced to be more reliant on transforming the image to match a stored canonical (upright) representation. The transform-then-match route is predicted to result in decreasingly efficient identification of increasingly disoriented stimuli, whereas no effect of orientation is predicted when an invariant-features route to recognition is used. Thus, a greater effect of orientation for trials with similar relative to dissimilar distractor alternatives would be predicated on the dual route account, since, as outlined above, subjects are likely to be forced to be more reliant on the orientation-sensitive transformthen-match route on similar distractor trials.

On the double-checking account, no effect of orientation on identification would be predicted for either distractor type. This is because, as specified above, the speed of response was irrelevant in the current task, and so, unlike in speeded naming tasks, subjects would not have been penalized if they had double-checked prior to responding.

\section{Method}

Subjects. There were 56 subjects. In both Experiments 1 and 2, subjects were from the University of Waterloo, Canada, and were paid to participate. In addition, subjects were native speakers of English, had normal or corrected-to-normal vision, and had not previously participated in picture recognition experiments.

Materials. The stimuli were 126 line drawings of familiar objects taken from Snodgrass and Vanderwart (1980). All the objects had an environmentally predominant orientation, which we labeled as $0^{\circ}$. Each drawing was rotated in steps of $30^{\circ}$ to give seven views of each object, from $0^{\circ}$ (upright) to $180^{\circ}$ (inverted). A pattern mask was produced, which was composed of small overlapping sections of a large number of different objects, none of which were presented in the experiment.

Design. Subjects completed two experimental blocks of 126 trials, each consisting of one view of each of the 126 objects. Each block was divided into three sub-blocks of 42 trials, and subjects could take a self-timed rest after each sub-block. On each trial, a picture of an object was presented at one of seven plane rotations $\left(0^{\circ}, 30^{\circ}, 60^{\circ}, 90^{\circ}, 120^{\circ}, 150^{\circ}\right.$, or $\left.180^{\circ}\right)$. The picture was followed by three written response alternatives, consisting of the name of the object presented and two distractor words (see Appendix). The distractor words named either two objects that were visually similar to the presented object ("similar" distractors), or two objects that were visually unrelated to the presented object ("dissimilar" distractors) The three response alternatives were labeled $1-3$. The presented object was labeled 1,2 , or 3 equally often; otherwise the ordering of response alternatives was random.

In each sub-block, six objects were presented at each orientation. Of these six objects, three were followed by similar distractors, and three were followed by dissimilar distractors. During the experiment, each subject saw two pictures of every object, once followed by similar distractors and once followed by dissimilar distractors. The order of presentation of trials within a sub-block was random and was different for every subject.

Twenty-eight subjects saw all pictures displayed for $28 \mathrm{msec}$, and the remaining 28 subjects saw all pictures displayed for $42 \mathrm{msec}$.
The exposure durations used were selected because they spanned the mid-range of possible durations. Duration had to be varied in multiples of the screen refresh time of $14 \mathrm{msec}$. Even at maximum stimulus contrast, performance was very poor at a 14-msec duration. Conversely, with a 56-msec duration, performance was so good that stimulus contrast had to be adjusted to extremely low levels and could not be varied smoothly to maintain subjects' overall performance at around $70 \%$ correct. We therefore investigated whether orientation influenced verification performance at either a 28 - or a 42-msec-exposure duration.

Across the 56 subjects, all items were depicted at all orientations an equal number of times. On half the trials, a given item was presented with similar distractors in the first block and dissimilar distractors in the second block of trials, and vice versa in the remaining trials.

Apparatus and Procedure. A PC-compatible 486 computer running the MEL Version 1.0 presentation package was used to display the stimuli. The experiment lasted about $30 \mathrm{~min}$.

The procedure for each experimental trial was as follows: A fixation cross appeared on the screen until the subject pressed the space bar. The fixation cross was then immediately replaced by a picture of an object, which was displayed for 28 or $42 \mathrm{msec}$ for the two different subject groups. The picture was replaced by a mask for $300 \mathrm{msec}$, which was in turn replaced by three written response alternatives, arranged vertically, and numbered 1-3. Subjects responded by pressing the appropriate key on the numeric keypad to select one of the three alternatives. The background screen was always white, and the fixation cross, picture, mask, and written response alternatives were gray or black. Subjects were not provided with feedback.

After each sub-block, the accuracy of the subject was calculated. If a subject was correct on $60 \%-80 \%$ of trials (where $33 \%$ correct was chance performance), the contrast of the pictures presented did not change for trials in the subsequent sub-block. However, if a subject was performing above $80 \%$ correct, the intensity of the picture was increased to a lighter shade of gray in the following sub-block, increasing the difficulty of the task by reducing the contrast of the picture relative to the white background. Conversely, if a subject was performing below $60 \%$ correct, the stimulus intensity was decreased to darken the stimulus in the subsequent sub-block, thereby increasing the contrast and making the picture easier to see. These measures were aimed at maintaining performance at around $70 \%$ correct, to maximize sensitivity to the experimental manipulations and to avoid floor and ceiling effects.

Subjects completed six practice blocks, each consisting of 14 trials, prior to the experimental blocks. Practice trials were identical to experimental trials except that different objects and distractor alternatives were presented. The practice blocks were used to set the appropriate initial level of stimulus contrast for a given subject. All subjects saw stimuli in the first practice block at the same contrast level. Contrast was adjusted from this point using a staircase algorithm that varied the size of the changes made to stimulus intensity throughout the experiment. Initially, intensity was adjusted in large steps, but step size decreased after each reversal in the staircase procedure.

Before the start of the practice trials, subjects completed three warm-up blocks, each consisting of five trials. Warm-up trials were identical to practice trials except that picture contrast was fixed at the initial maximum contrast level and the picture presentation duration varied. For the 28 subjects presented with pictures for $28 \mathrm{msec}$ in the experimental blocks, pictures were presented for 400,100 , and $42 \mathrm{msec}$ in the first, second, and third warm-up blocks, respectively. For the 28 subjects presented with pictures for $42 \mathrm{msec}$ in experimental blocks, pictures were presented for 700,400 , and $100 \mathrm{msec}$ in the first, second, and third warm-up blocks, respectively.

Subjects were instructed that speed of response was irrelevant to the task. Subjects were also told that the contrast of the stimuli would vary throughout the experiment, and the reason for this vari- 
ation was explained. Finally, subjects were encouraged to perform as accurately as possible, even if the stimulus became difficult to see, which could happen as a rest $t$ of the staircase procedure.

\section{Results}

No subjects were replaced. An analysis of variance (ANOVA) was conducted on the mean percent correct verification responses. Mean percent correct responses over subjects are shown in Figure 1 and Table 1 . The difference between the mean percent correct verification over subjects in the dissimilar and similar conditions is shown in Figure 2. There were three within-subjects factors: orientation (the plane rotation of the picture $-0^{\circ}$, $30^{\circ}, 60^{\circ}, 90^{\circ}, 120^{\circ}, 150^{\circ}$, or $180^{\circ}$ ), distractor (the visual similarity of the presented object to the object named by the distractor words-similar or dissimilar), and block (the block in which the picture was seen-1 or 2). There was one between-subjects factor, duration (the presentation duration of the picture- 28 or $42 \mathrm{msec}$ ).

The main effect of orientation was significant $[F(6,324)$ $=33.01, p<.001$, as was that of distractor $[F(1,54)=$ $998.73, p<.001]$. Subjects were more accurate on dissimilar distractor trials $(79.3 \%)$ than on similar distractor trials $(61.5 \%)$. Duration was significant $[F(1,54)=$ $7.10, p<.02]$. Subjects were more accurate at the $28-\mathrm{msec}$

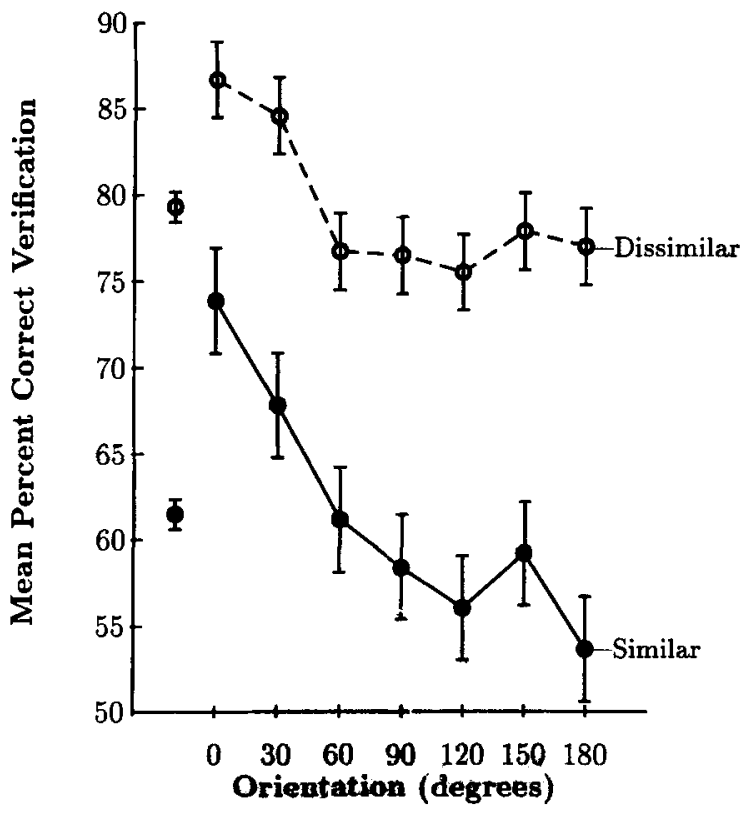

Figurc 1. Resifts of Experiment 1. Maen pereent correct verification for ench orientation in the similar and dissimllar condilions, along with the $9 \%$ conihdemee interval based on the error ierm tor the orientation effect th the within-subjects ANOVA. These confidence intervals can be used to evafuate the reliability of the oriantation effects in this experiment. The two lofinest, disconnected, data points are the means for the similar and diasimilar conditions averaged across orientation, along with $95 \%$ confidence intervals based on the error term for the similarity factor in the within-subjects ANOVA.
Table 1

Mean Percent Correct Verification Rates as a Function of Orientation, Stimulus Duration, and Presentation of Similar or Dissimilar Distractors in Experiment 1

\begin{tabular}{|c|c|c|c|c|c|c|c|c|}
\hline \multirow[b]{2}{*}{ Condition } & \multicolumn{7}{|c|}{ Orientation } & \multirow[b]{2}{*}{ Mear } \\
\hline & $0^{\circ}$ & $30^{\circ}$ & $60^{\circ}$ & $90^{\circ}$ & $120^{\circ}$ & $150^{\circ}$ & $180^{\circ}$ & \\
\hline \multicolumn{9}{|c|}{ 28-msec stimulus duration } \\
\hline Similar distractor & 79 & 70 & 62 & 60 & 55 & 59 & 55 & 63 \\
\hline Dissimilar distractor & 91 & 88 & 79 & 81 & 78 & 81 & 80 & 82 \\
\hline \multicolumn{9}{|c|}{ 42-msec stimulus duration } \\
\hline Similar distractor & 69 & 66 & 61 & 57 & 57 & 60 & 53 & 60 \\
\hline Dissimilar distractor & 83 & 81 & 75 & 72 & 73 & 75 & 74 & 76 \\
\hline Overall means & 80 & 76 & 69 & 68 & 66 & 69 & 65 & 70 \\
\hline
\end{tabular}

stimulus duration $(72.6 \%)$ than at the $42-\mathrm{msec}$ stimulus duration $(68.25 \%)$, indicating that the staircase algorithm slightly overcompensated for differences in the difficulty of identifying stimuli at the different stimulus durations. The main effect of block was not significant $[F(1,54)=$ $.54, p>.4]$.

The orientation $\times$ distractor interaction was significant $[F(6,324)=3.23, p<.005]$. The identification benefit for $0^{\circ}$ and $30^{\circ}$ near upright views relative to views disoriented by $60^{\circ}$ or more was greater for similar distractors $(13.2 \%)$ than for dissimilar distractors $(8.9 \%$; see Figures 1 and 2). The distractor $\times$ duration interaction was also significant $[F(1,54)=10.96, p<.002]$. The difference between similar and dissimilar distractors was greater at the $28-\mathrm{msec}$ picture duration $(19.7 \%)$ than at the 42 -msec duration $(16.0 \%)$. Neither the orientation $X$ block $[F(6,324)=.20, p>.9]$ nor the orientation $\times$ duration $[F(6,324)=1.78, p>.1]$ interaction was significant, and there were no other significant interactions.

\section{Discussion}

Clearly, plane disorientation influenced performance in verifying briefly presented masked pictures of objects. Upright views were identified more accurately than $30^{\circ}$ views, which in turn were verified more accurately than $60^{\circ}$ views. However, performance was similar across orientations that were rotated further from upright, especially for the dissimilar distractor condition. The verification of $0^{\circ}$ and $30^{\circ}$ views was around $10 \%$ more accurate than the verification of more disoriented views. We will term this identification benefit for upright and near upright views relative to more severely disoriented views (rotated $60^{\circ}$ or more from the canonical upright orientation) the near upright orientation effect.

Thus in Experiment 1, a clear orientation effect was observed in a task in which double-checking (Corballis, 1988; De Caro \& Reeves. 1995) could not have influenced performance. The results suggest to us that the representations mediating object recognition must be orientation sensitive. Orientation-sensitive representations suggest the need for matching procedures that can compensate for differences in orientation between the represented view stored in memory and the view present at input during the 


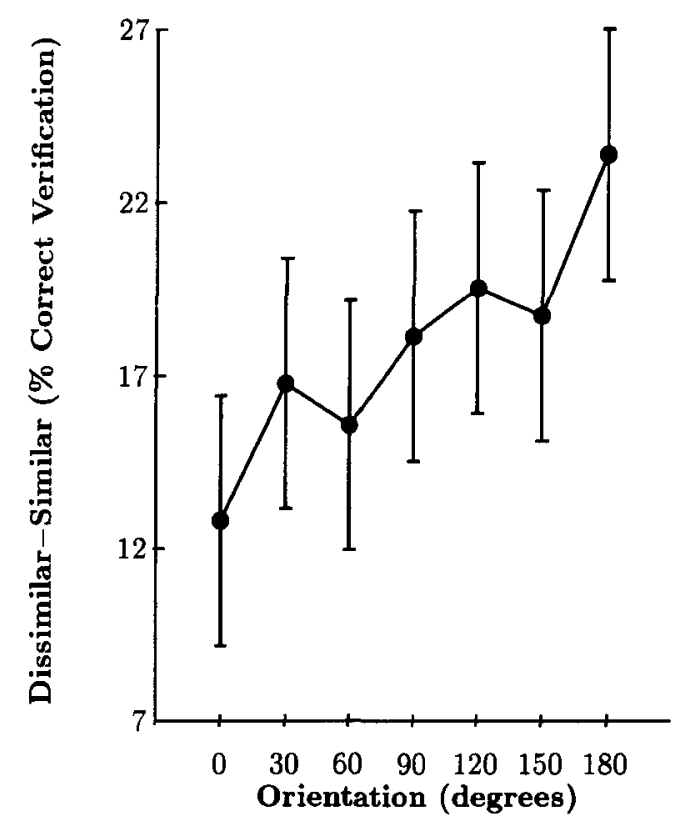

Figure 2. Results of Experiment 1. Difference between mean percent correct verification in the dissimilar condition and the similar condition, for each orientation, along with the $95 \%$ confidence interval of the difference score, based on the error term for the similarity $x$ orientation interaction effect in the withinsubjects ANOVA. These confidence intervals can be used to evaluate the reliability of the difference in the orientation effects across the similar and dissimilar conditions. This figure illustrates more directly the nature, form, and reliability of the interaction between orientation and similarity displayed in Figure 1.

recognition process. This in turn makes it more likely that such processing was also a major factor, or was the sole factor, in causing the orientation effects observed in speeded naming tasks (e.g., Jolicoeur, 1985).

Not surprisingly, the form of the function relating accuracy of verification to orientation of the object in the present paradigm differed from that observed on response time in speeded naming tasks (e.g., De Caro \& Reeves, 1995; Jolicoeur, 1985; Jolicoeur \& Milliken, 1989; McMullen \& Jolicoeur, 1990, 1992). Especially for the dissimilar distractor condition, there was almost a plateau in performance over the range of plane rotations from $60^{\circ}$ to $150^{\circ}$, in contrast to a smooth, almost linear increase in the latency of picture naming found over this range. This difference across paradigms is to be expected if the short exposure duration followed by immediate masking of the stimuli in Experiment 1 does not allow enough time for a time-consuming continuous normalization process to operate. Thus, the transform-then-match route is likely to be unsuccessful, except when little or no image transformation is required before the stimulus can be matched to a stored orientation-specific representation. Orientationsensitive stored representations will generally represent only commonly experienced views, such as upright views.
Thus only upright and near upright views will benefit from identification via the transform-then-match route when the presentation conditions limit the time available to normalization processes.

If the transform-then-match route to recognition fails (as it will for most highly disoriented views of objects), then on the dual route account (Jolicoeur, 1990), subjects will be forced to rely on the alternative invariant-features route to recognition. Thus, in the present paradigm, for views that cannot be processed successfully by the transformthen-match route, one would expect little or no effect of orientation. Furthermore, as discussed in the introduction to Experiment 1, the invariant-features route is predicted to be more successful when orientation-invariant features could readily be identified, as in the dissimilar distractor condition. As expected, the manipulation of the visual similarity of the presented object to the distractor alternatives had a strong influence on performance. Verification was more difficult for visually similar than for dissimilar distractors. In addition, and more interestingly, the orientation effect was larger for similar than for dissimilar distractors (Figure 2). The results are consistent with the view that a reduced orientation effect would be observed when dissimilar distractors were presented because it would be easier to find orientation-invariant features that could uniquely identify a picture on such trials, and subjects would then be less reliant on the orientationsensitive transform-then-match route to recognition. This issue was examined further in Experiment 2.

Finally, there was no indication of a reduction in the magnitude of the orientation effect from the first to the second presentation of an object, from Block 1 to Block 2. The near upright orientation effect was $10.7 \%$ in Block 1 and $11.3 \%$ in Block 2 . This contrasts with the results of picture naming studies, which have shown clear reductions in the size of orientation effects, especially from the first to the second identification of an object (Jolicoeur, 1985; Jolicoeur \& Milliken, 1989; Murray et al., 1993). In Experiment 2, we examined this issue further.

One motivation that might lead us to postulate that the orientation effects observed in speeded naming tasks were produced by occasional double-checking is the hypothesis that the representations that mediate object recognition encode shape using an orientation-free code (Corballis, 1988). The results of Experiment 1, which revealed clear effects of plane rotation on the identification of briefly masked pictures, however, support the claim that the representations of objects mediating recognition are orientation sensitive. In our view, this fact removes the motivation to postulate a special epiphenomenal explanation, such as double-checking (Corballis, 1988; De Caro \& Reeves, 1995), for the increase in naming time observed when subjects perform speeded naming of rotated objects (see, e.g., Jolicoeur, 1985). Instead, we propose that it is more parsimonious simply to suppose that the orientation sensitivity that we demonstrated in Experiment 1, 
which cannot be accounted for by double-checking, is also the basic cause of the orientation effects observed in speeded naming experiments.

\section{EXPERIMENT 2}

In Experiment 1, there was an interaction between the effects of orientation and distractor type, with a larger orientation effect for similar than for dissimilar distractor trials. The near upright orientation effect was $13.2 \%$ with similar distractors and $8.9 \%$ with dissimilar distractors. However, performance with the dissimilar distractors was also much better than performance with the similar distractors. We conducted Experiment 2 to try to equate performance across distractor type by using a staircase algorithm to adjust stimulus contrast independently for similar and dissimilar distractor trials. This allowed us to examine whether the orientation effect across the two distractor types still differed when mean performance for similar and dissimilar distractors was approximately equal.

In addition, Experiment 2 examined in more detail the effect of practice on orientation effects. The results of Experiment 1 suggested that there was no interaction between the magnitude of orientation effects and whether a subject had already seen a given picture in the experiment. In contrast, a number of picture naming studies have revealed significant reductions in the size of orientation effects with practice, particularly from the first to the second presentation of a given object (e.g., Jolicoeur, 1985; Jolicoeur \& Milliken, 1989; Murray et al., 1993). However, in typical picture naming studies, most objects are identified correctly on every presentation. In contrast, in Experiment 1, there were high error rates, and furthermore, subjects had a $33 \%$ chance of correctly guessing the identity of an object on a trial. The mean rate of correct picture identification was $70 \%$ correct in Block 1 , of which around $15 \%$ of responses can be assumed to be correct guesses. We can thus estimate that subjects actually verified only around $55 \%$ of objects in the first block. Even on trials in which subjects did genuinely verify the identity of the object, they may often have had access to only a degraded percept, relative to that in a typical naming experiment, in which the stimulus is displayed until subjects respond. Such a degraded percept may have been adequate to discriminate the object presented from two distractor alternatives (particularly for dissimilar distractor trials), but this information may often have been insufficient to uniquely identify the object.

Since little or no reduction in orientation effects would be predicted for objects that were not correctly identified in the first block (for objects presented on error trials, trials in which subjects guessed the correct response, or trials in which subjects had access to only a highly degraded percept), any reduction in orientation effects from Block 1 to Block 2 would be expected to be diluted in Experiment 1 relative to a typical naming task. Indeed, a reduction in view effects with practice might not become apparent in the masking task without a larger number of repetitions of each object. In Experiment 2, we doubled the number of presentations of each object for a given subject from two, as in Experiment 1, to four.

\section{Method \\ Subjects. There were 56 subjects.}

Materials. The stimuli presented in Experiment 1 were used.

Design. The design was identical to that of Experiment 1 except for the following differences: Subjects completed four experimental blocks of 126 trials, each consisting of one view of each of the 126 objects. During the experiment, each subject saw four pictures of every object, twice followed by similar distractor alternatives, and twice followed by dissimilar distractor alternatives.

Apparatus and Procedure. The apparatus was the same as in Experiment 1. The procedure was identical to that of Experiment 1 except for the following differences: Stimulus contrast was adjusted independently for similar and dissimilar distractor trials to try to equate performance levels across distractor types. Contrast was varied using the same staircase algorithm as in Experiment 1. After each sub-block, the accuracy of a subject on the 21 similar distractor trials and the 21 dissimilar distractor trials was calculated separately, and the stimulus intensity for the following sub-block was set independently for similar and dissimilar distractor trials. There were four practice blocks, each consisting of 28 trials, with 14 similar and 14 dissimilar distractor trials. The experiment lasted about $45 \mathrm{~min}$.

\section{Results}

Eleven subjects, all of whom had seen pictures presented for $28 \mathrm{msec}$, were replaced in Experiment 2 because

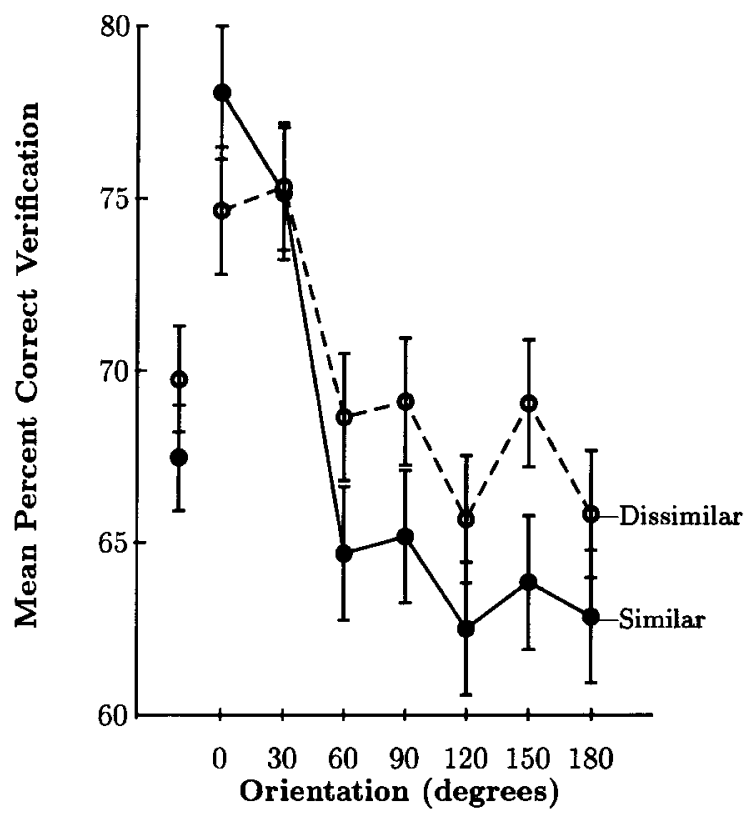

Figure 3. Results of Experiment 2. Mean percent correct verification for each orientation in the similar and dissimilar conditions, along with the $95 \%$ confidence interval based on the error term for the orientation effect in the within-subjects ANOVA. These confidence intervals can be used to evaluate the reliability of the orientation effects in this experiment. The two leftmost, disconnected, data points are the means for the similar and dissimilar conditions, averaged across orientation, along with $95 \%$ confidence intervals based on the error term for the similarity factor in the within-subjects ANOVA. 
Table 2

\begin{tabular}{|c|c|c|c|c|c|c|c|c|}
\hline \multirow[b]{2}{*}{ Condition } & \multicolumn{7}{|c|}{ Orientation } & \multirow[b]{2}{*}{ Mean } \\
\hline & $0^{\circ}$ & $30^{\circ}$ & $60^{\circ}$ & $90^{\circ}$ & $120^{\circ}$ & $150^{\circ}$ & $180^{\circ}$ & \\
\hline \multicolumn{9}{|l|}{ 28-msec stimulus duration } \\
\hline Similar distractor & 81 & 79 & 67 & 68 & 64 & 66 & 64 & 70 \\
\hline Dissimilar distractor & 74 & 75 & 68 & 68 & 65 & 68 & 65 & 69 \\
\hline \multicolumn{9}{|c|}{ 42-msec stimulus duration } \\
\hline Similar distractor & 75 & 72 & 62 & 63 & 61 & 62 & 62 & 65 \\
\hline Dissimilar distractor & 75 & 76 & 69 & 70 & 66 & 70 & 67 & 70 \\
\hline Overall means & 76 & 75 & 67 & 67 & 64 & 66 & 64 & 69 \\
\hline
\end{tabular}

stimulus intensity dropped to zero (i.e., minimum stimulus intensity, and maximum stimulus contrast relative to the background) for the similar distractor trials for the final sub-blocks for these subjects. This meant that for these subjects, the staircase algorithm could not compensate any further to try to equate the difficulty of similar and dissimilar distractor trials by adjusting stimulus intensity.

An ANOVA was conducted on the mean percent correct verification responses. Mean percent correct responses over subjects is shown in Figure 3 and Table 2. The difference between the mean percent correct verification over subjects in the dissimilar and similar conditions is shown in Figure 4. There were three within-subjects factors: orientation (the plane rotation of the picture $-0^{\circ}, 30^{\circ}$, $60^{\circ}, 90^{\circ}, 120^{\circ}, 150^{\circ}$, or $180^{\circ}$ ), distractor (the visual similarity of the presented object to the object named by the distractor words - similar or dissimilar), and block (the block in which the picture was seen-1,2,3, or 4). There was one between-subjects factor, duration (the presentation duration of the picture- $28 \mathrm{msec}$ or $42 \mathrm{msec}$ ).

The main effect of orientation was significant $[F(6,324)$ $=56.19, p<.001]$, as was that of distractor $[F(1,54)=$ $5.08, p<.03$ ]. Subjects were more accurate for dissimilar distractors $(69.8 \%)$ than for similar distractors $(67.5 \%)$. The main effects of duration $[F(1,54)=1.96, p>.1]$ and for block $[F(3,162)=1.51, p>.2]$ were not significant.

The orientation $\times$ distractor interaction was significant $[F(6,324)=4.55, p<.001]$. The near-upright orientation effect was greater for similar distractors $(12.8 \%)$ than for dissimilar distractors (7.3\%; see Figures 3 and 4$)$.

The distractor $\times$ block interaction was significant $[F(3,162)=13.53, p<.001]$. Similar distractors were verified $9.2 \%$ and $0.9 \%$ less accurately than dissimilar distractor in Blocks 1 and 2 , respectively, and $0.2 \%$ and $0.8 \%$ more accurately in Blocks 3 and 4 , respectively. The distractor $\times$ duration interaction was also significant $[F(3,162)=8.31, p<.006]$. Similar distractors were verified $0.6 \%$ more accurately than dissimilar distractors at the $28-\mathrm{msec}$ duration, and $5.2 \%$ less accurately at the 42 -msec duration. The latter two interactions were not of interest here and were probably a result of variation in the success of the staircase algorithm in compensating for differences in the ease of verification of stimuli presented at different durations and with different distractor types over the course of the experiment.

The orientation $\times$ block interaction was marginally significant $[F(18,972)=1.58, p<.06]$. The near upright orientation effect did reduce a little over Blocks 1-4, with the effect being $10.4 \%$ in Block $1,11.5 \%$ in Block 2, $10.2 \%$ in Block 3, and $8.1 \%$ in Block 4 . However, there was no indication of an interaction between the linear component of the orientation effect and block $[F(1,54)=1.19, p>.2]$. Instead, this marginal interaction was probably due largely to the results of Block 2 , in which, in contrast to the other blocks, subjects were more accurate at verifying $30^{\circ}$ views than $0^{\circ}$ views. The orientation $\times$ duration interaction $[F(6,324)=0.60, p>$ .7] was not significant, and no other interactions were significant.

\section{Discussion}

The results of Experiment 2 generally replicated those of Experiment 1. There was a clear orientation effect, in which, as in Experiment 1, most of the effects occurred between $0^{\circ}$ and $60^{\circ}$. Furthermore, as in Experiment 1, there was a significant orientation $\times$ distractor interaction (Figure 4), with a larger orientation effect for similar distractors than for dissimilar distractors. However,

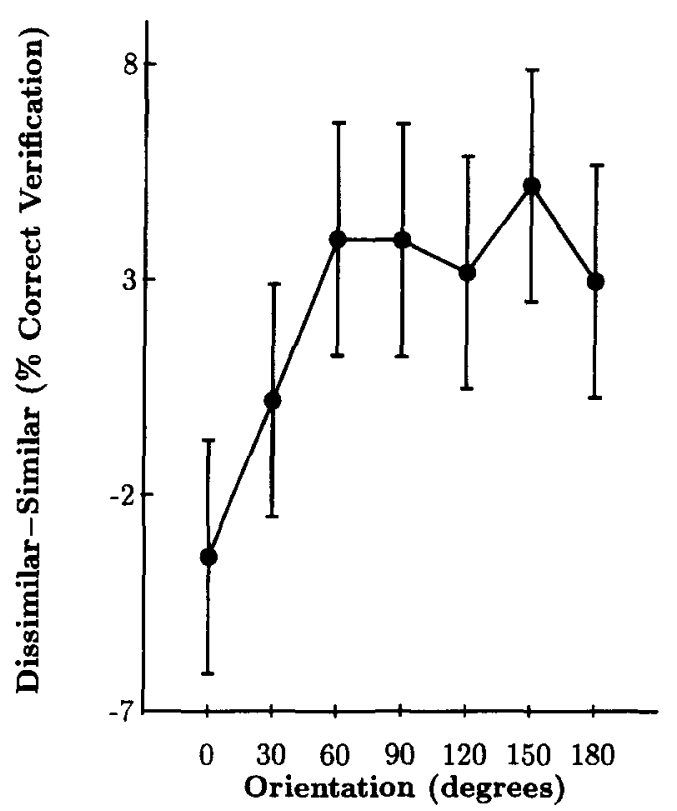

Figure 4. Results of Experiment 2. Difference between mean percent correct verification in the dissimilar condition and the similar condition, for each orientation, along with the $95 \%$ confidence interval of the difference score, based on the error term for the similarity $x$ orientation interaction effect in the withinsubjects ANOVA. These confidence intervals can be used to evaluate the reliability of the difference in the orientation effects across the similar and dissimilar conditions. This figure illustrates more directly the nature, form, and reliability of the interaction between orientation and similarity displayed in Figure 3. 


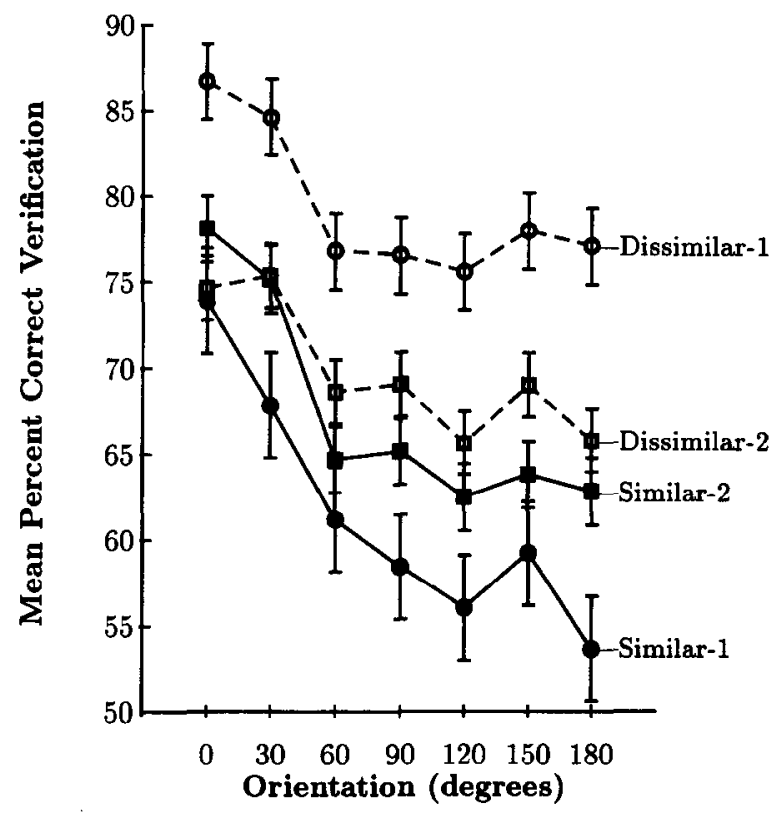

Figure 5. Results of Experiments 1 and 2. Mean percent correct verification for Experiment 1 (circles) and Experiment 2 (squares), for each orientation in the similar and dissimilar conditions, along with the $95 \%$ confidence interval based on the error term for the orientation effect in the separate within-subjects ANOVAs. This figure replots, on the same scale, the key results from Figures 1 and 3 and illustrates the similarity of the orientation $\times$. similarity interaction across the two experiments. This figure also shows how well the staircase algorithm worked in bringing the performance across similar and dissimilar trials into the same range.

in contrast to the findings from Experiment 1, there was little overall difference in performance accuracy for similar and dissimilar distractor trials because stimulus contrast was adjusted to equate performance across these two conditions. As can be seen in Figure 5, the contrastadjustment procedure was successful in equating performance across trials with dissimilar and similar distractor alternatives. Thus the orientation $\times$ distractor interaction cannot be explained as an artifact due to a ceiling effect for responses for the dissimilar distractor trials. Instead, this result supports our proposal that a reduced orientation effect is observed when dissimilar rather than similar distractors are presented. We argue that this effect arises because, on dissimilar distractor trials, subjects are more likely to be successful in encoding orientation-invariant features that can distinguish between the response alternatives, and subjects are thus less reliant on matching to orientation-specific information.

As in Experiment 1, there was little evidence that the orientation effect decreased with practice, although the effect was somewhat smaller in the final block. This result is discussed further below.

\section{GENERAL DISCUSSION}

The results of Experiments 1 and 2 were clear-cut: The identification of objects, as assessed by an unspeeded, delayed, forced-choice discrimination, was systematically reduced as the orientation of the stimuli deviated further from a canonical upright view. These effects were highly statistically significant and were found in all the conditions examined (varying exposure duration, distractor type, and number of presentations of an object). Upright and near upright views were verified more accurately than more highly disoriented views, indicating that object representations are not orientation invariant. These results were obtained in a paradigm in which doublechecking (Corballis, 1988; De Caro \& Reeves, 1995) could not influence performance. The results provide strong empirical support for the claim that the representations that mediate object recognition are sensitive to viewpoint, and in particular to in-plane orientation.

Corballis (1988) proposed that visual shape might be encoded by representations that used orientation-free coding. Although he suggested that there may be different types of representations, some orientation free and some orientation sensitive, the main thrust of his article (we believe) was to provide arguments and evidence in support of orientation-free coding in the mediation of object recognition. From this point of view, the results of experiments demonstrating large and systematic effects of orientation on the time to identify rotated objects (e.g., Jolicoeur, 1985) might appear to be problematic. If shapes are represented using orientation-free codes, and if these representations are those that dominate object recognition, then one would not expect to find any orientation effects in tasks that depend on recognition (such as naming). To deal with this problem, Corballis suggested that one could explain the orientation effect on naming time if subjects occasionally used mental rotation to doublecheck an initial stimulus identification that had been derived by finding a match to an orientation-free representation in memory. This possibility, of course, assumes that there are representations that are orientation sensitive to which one can mentally rotate the representation of an object that has already been recognized (perhaps partially), but is in need of double-checking. Corballis postulated the existence of canonically oriented representations in order to account for the results of experiments in which the task required a left-right or mirror-normal discrimination ("mental-rotation" experiments). As we mentioned earlier (Experiment 1, Discussion), we believe that the main motivation for entertaining double-checking as an account of orientation effects on naming time was the hypothesis that recognition is usually achieved by activating orientation-free representations. The present results, however, provide unambiguous evidence for the importance of orientation-sensitive representations in the process of object recognition, at least under the present testing conditions.

Our results do not allow us to rule out that doublechecking might be taking place in experiments using speeded naming paradigms. However, we believe that our results render the double-checking account less appealing and less parsimonious. Given that we have clearcut evidence for recognition mediated by orientation- 
sensitive representations in the present paradigm, it seems to us more parsimonious to assume that the same type of representation is involved when subjects perform speeded naming. There is no need to argue that subjects epiphenomenally double-check identifications that they have already obtained by matches to orientation-free representations. We believe that our arguments are strengthened by the presence of large orientation effects even when the distractors were very dissimilar to the target object. We believe that, in this case, we have given every opportunity for potential orientation-free representations to dominate performance. Nonetheless, orientation-sensitive representations play an obvious role in recognition even when the task is to discriminate among DEER, BOWL, and BOW.

Thus, although our results cannot rule out the potential involvement of double-checking in the cause of the orientation effects observed in speeded naming tasks, we argue that the present findings put the onus on proponents of the double-checking hypothesis to demonstrate the necessity of this additional account of some orientation effects. Instead, we suggest that the effects of plane disorientation observed in both speeded and unspeeded tasks occur because canonically oriented objects are matched more efficiently to stored orientation-sensitive representations than are highly disoriented stimuli.

The orientation effects that we have reported were not identical to those typically obtained from picture naming studies presenting similar stimuli. First, in previous picture naming studies, plane rotation effects have been found to decrease with increasing experience with stimuli, with the greatest reduction in orientation effects occurring from the first to the second presentation of the stimuli (Jolicoeur, 1985; Jolicoeur \& Milliken, 1989). However, in Experiments 1 and 2, there was no evidence for a decrease in the near upright orientation effect across blocks except for a nonsignificant trend for a decrease in Block 4 of Experiment 2. This difference is probably due to task changes across naming and masking studies, since a similar pattern of results has been observed in studies that have required the identification of alphanumeric stimuli. Corballis, Zbrodoff, Shetzer, and Butler (1978) reported a rapid reduction with practice in the initial (small) orientation effects found for naming disoriented characters, whereas Jolicoeur and Landau (1984) reported no reduction with practice in the strong effects of plane disorientation on the identification of alphanumeric characters that were briefly presented and masked.

Second, in previous picture naming studies, naming latencies have been reported to increase approximately linearly for plane disorientations across the range of $0^{\circ}$ to $150^{\circ}$ (e.g., De Caro \& Reeves, 1995; Jolicoeur, 1985; Jolicoeur \& Milliken, 1989; McMullen \& Jolicoeur, 1990, 1992). In contrast, in Experiments 1 and 2, there was a strong effect of plane disorientation over the range of $0^{\circ}$ to $60^{\circ}$ but only a weak effect over the range of $60^{\circ}$ to $150^{\circ}$. However, there was still a significant effect of plane dis-

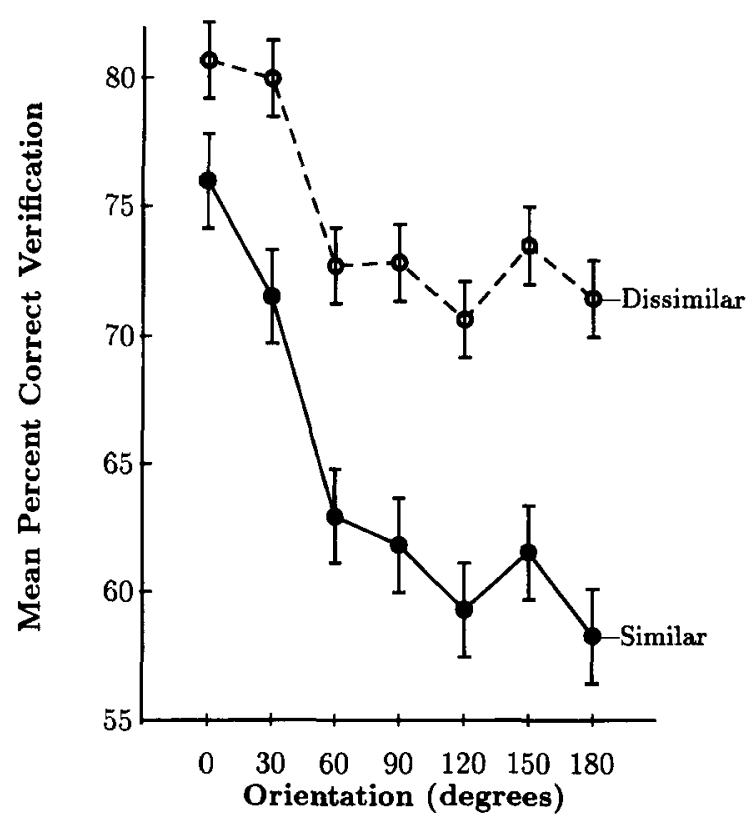

Figure 6. Results of Experiments 1 and 2. Mean percent correct verification, for the combined data from Experiments 1 and 2, for each orientation in the similar and dissimilar conditions, along with the $95 \%$ confidence interval based on the error term for the orientation effect.

orientation over the range of $60^{\circ}$ to $150^{\circ}$ for the similar distractor trials (see Figure 6).

The overall results can be accounted for by the dual route account of the effects of plane rotation proposed by Jolicoeur (1990). This account suggests that there are two distinct routes to object recognition-a transformthen-match route and an invariant-features route.

In the transform-then-match route, an image is first transformed to normalize it, before it is matched to a stored orientation-sensitive representation. More disoriented images will require more time to be normalized and therefore will be identified less efficiently. We propose that a reasonably good, stable internal representation is required if the transformation procedure is to be successful. The transform-then-match route to identification was therefore often unavailable under the conditions of the present Experiments 1 and 2, which presented very brief, faint, and immediately masked pictures of objects. The images of these pictures would be too unstable to undergo a major transformation in order to be accurately normalized to a canonical upright orientation.

However, the image transformation stage in the transform-then-match route could be completed very rapidly or might not be required at all when an upright or near upright view was presented, which was already at or close to the canonical orientation of the object. These views could then be matched efficiently and fairly directly to stored, orientation-specific (canonical, upright) 


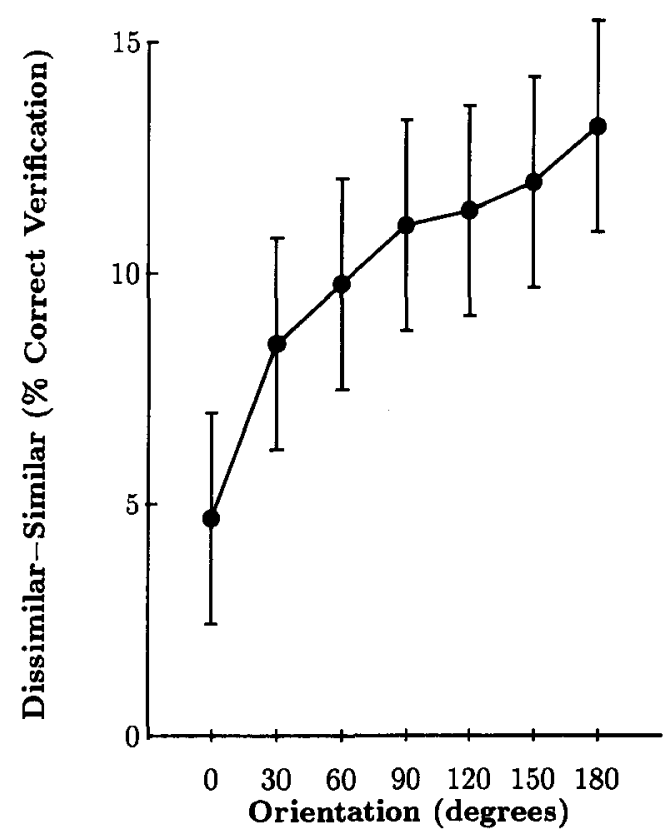

Figure 7. Results of Experiments 1 and 2. Difference between mean percent correct verification in the dissimilar condition and the similar condition, for the combined data from Experiment 1 and the first two blocks of Experiment 2, for each orientation, along with the $95 \%$ confidence interval based on the error term for the orientation effect.

representations, with minimal image normalization being required.

Such relatively direct matching could not, however, mediate accurate identification of more disoriented views of objects. Instead, for such views, recognition must be mediated by the alternative to the transform-then-match route- the invariant-features route. In the latter route, distinctive orientation-invariant features are extracted from the image and are matched directly to stored orientationinvariant representations. This route is generally not as efficient as the transform-then-match route (otherwise, no orientation effects on verification would have been observed), but it was the only route available to verify the highly disoriented views of objects presented in Experiments 1 and 2 .

We can use this dual route account of the effects of plane rotation on the identification of familiar objects to explain the results of Experiments 1 and 2. For upright or near-upright views of objects, subjects could use the efficient transform-then-match route. This would be particularly useful when visually similar distractor alternatives were presented (Figure 7). This is because the alternative route to recognition, the invariant-features route, is relatively inefficient when an object must be discriminated from among visually similar distractors, since it is then difficult to extract a simple orientation- invariant distinguishing feature that is unique to the presented object.

However, we should note that even for $0^{\circ}$ and $30^{\circ}$ views, subjects were still much better at identifying objects when dissimilar rather than similar distractors were presented. This is not a problem for our account-an object will not always be correctly identified, even if the transform-thenmatch route can be used. Any mismatches via this route will likely activate a representation of a visually similar object. This might still allow the accurate identification of the object presented from among visually dissimilar distractors, but not from visually similar distractors. For example, if a subject was given three visually dissimilar response alternatives-BARN, HORSE, and BICYCLE-and the subject mistakenly believed that a picture of a dog had been presented, the subject could make an informed guess that a picture of a horse had actually been presented. However, if the subject was given three visually similar response alternatives-DONKEY, HORSE, and cOW-the subject would probably be forced to make a random choice among the alternatives.

\section{REFERENCES}

Corballis, M. C. (1988). Recognition of disoriented shapes. Psychological Review, 95, 115-123.

Corballis, M. C., Zbrodoff, N. J., Shetzer, L. I., \& Butler, P. B. (1978). Decisions about identity and orientation of rotated letters and digits. Memory \& Cognition, 6, 98-107.

De Caro, S. A., \& ReEves, A. (1995, November). Object recognition without mental rotation. Paper presented at the annual meeting of the Psychonomic Society, Los Angeles.

JOLICOEUR, P. (1985). The time to name disorientated natural objects. Memory \& Cognition, 13, 289-303.

JoLicoeur, P. (1990). Identification of disoriented objects: A dual systems theory. Mind \& Language, 5, 387-410.

JOLICOEUR, P., \& LANDAU, M. J. (1984). Effects of orientation on the identification of simple visual patterns. Canadian Journal of Psychology, 38, 80-93.

JOLICOEUR, P., \& MILLIKEN, B. (1989). Identification of disoriented objects: Effects of context of prior presentation. Journal of Experimental Psychology: Learning, Memory, \& Cognition, 15, 200-210.

MARR, D. (1982). Vision. San Francisco: W. H. Freeman.

McMullen, P. A., \& Jolicoeur, P. (1990). The spatial frame of reference in object naming and discrimination of left-right reflections. Memory \& Cognition, 18, 99-115.

MCMULLEN, P. A., \& Jolicoeur, P. (1992). The reference frame and effects of orientation on finding the top of rotated objects. Journal of Experimental Psychology: Human Perception \& Performance, 18, 807-820.

MURRAY, J. E. (1995). The role of attention in the shift from orientationdependent to orientation-invariant identification of disoriented objects. Memory \& Cognition, 23, 49-58.

Murray, J. E., Jolicoeur, P., McMullen, P. A., \& Ingleton, M (1993). Orientation-invariant transfer of training in the identification of rotated natural objects. Memory \& Cognition, 2, 604-610.

SNODGRASS, J. G., \& VANDERWART, M. (1980). A standardized set of 260 pictures: Norms for name agreement, image agreement, familiarity and visual complexity. Journal of Experimental Psychology: Human Perception \& Performance, 6, 174-215.

UlLman, S. (1989). Aligning pictorial descriptions: An approach to object recognition. Cognition, 32, 193-254. 
APPENDIX

List of the 126 Target Pictures Presented, Together With the Two Written Distractor Alternatives Presented on Visually Similar and Visually Dissimilar Trials in Experiments 1 and 2

\begin{tabular}{|c|c|c|c|c|}
\hline \multirow{3}{*}{$\begin{array}{c}\text { Target } \\
\text { Picture } \\
\text { Presented } \\
\text { Airplane }\end{array}$} & \multicolumn{4}{|c|}{ Written Distractors } \\
\hline & \multicolumn{2}{|c|}{ Visually Similar Items } & \multicolumn{2}{|c|}{ Visually Dissimilar Items } \\
\hline & Arrow & Rocket & Cow & Garbage-Can \\
\hline Alligator & Snake & Lizard & Watch & Baby-Carriage \\
\hline Ant & Spider & Beetle & Cart & Donkey \\
\hline Apple & Tomato & Peach & Horse & Weighing-Scales \\
\hline Baby-Carriage & Wagon & Shopping Cart & Bucket & Cat \\
\hline Barn & House & Church & Ladle & Porcupine \\
\hline Basket & Crate & Rug & Fish & Dog \\
\hline Bear & Dog & Cow & Gate & Orange \\
\hline Bed & Couch & Table & Snake & Bus \\
\hline Bee & Fly & Ant & Chair & Cauliflower \\
\hline Beetle & Spider & Ant & Train & Coat \\
\hline Bicycle & Motorcycle & $\mathrm{Car}$ & Shoe & Nail \\
\hline Bird & Mouse & Bat & Sweater & Spinning-Wheel \\
\hline Blouse & Sweater & Coat & Mouse & Truck \\
\hline Boot & Shoe & Sock & Snake & Fox \\
\hline Bottle & Jar & Vase & Hat & Necklace \\
\hline Bowl & Cup & Plate & Trolley & Table \\
\hline Bus & Trolley & Truck & Cup & Goose \\
\hline Cake & Hat & Cheese & Horse & Barn \\
\hline Camel & Horse & Cow & Doorknob & Beetle \\
\hline Candle & Doorknob & Nail & Jar & Peach \\
\hline Cannon & Cart & Spinning-Wheel & Spider & Chair \\
\hline Car & Trailer & Truck & Dog & Shirt \\
\hline Cat & Dog & Fox & Trailer & Merry-Go-Round \\
\hline Caterpillar & Flute & Necklace & Stool & Pitcher \\
\hline Chair & Stool & Table & Flute & Donkey \\
\hline Chicken & Turkey & Goose & House & Puppet \\
\hline Church & House & Barn & Turkey & Deer \\
\hline Clock & Watch & Weighing-Scales & Tomato & Couch \\
\hline Coat & Night-gown & Shirt & Desk & Pigeon \\
\hline Couch & Desk & Chair & Night-gown & Mammoth \\
\hline Cow & Horse & Donkey & Cake & Desk \\
\hline Crown & Cake & Merry-Go-Round & Horse & Goose \\
\hline Cup & Bowl & Pitcher & Goat & Cherry \\
\hline Deer & Goat & Donkey & Bowl & Bow \\
\hline Desk & Drawers & Couch & Goat & Boot \\
\hline Dog & Goat & Deer & Drawers & Beetle \\
\hline Doll & Clown & Puppet & Cow & Spoon \\
\hline Donkey & Cow & Horse & Clown & Basket \\
\hline Dress & Bell & Bow & Toaster & Cat \\
\hline Dresser & Toaster & Desk & Bell & Turtle \\
\hline Duck & Swan & Goose & Sock & Dinosaur \\
\hline Eagle & Owl & Pigeon & Buffalo & Glass \\
\hline Elephant & Buffalo & Mammoth & Propeller & Beetle \\
\hline Flower & Propeller & Cherry & Swan & Garbage-Can \\
\hline Fly & Bee & Beetle & Owl & Dog \\
\hline Foot & Sock & Boot & Bee & Toddler \\
\hline Football-Helmet & Basket & Bear-Trap & Dog & Pillar \\
\hline Fox & Dog & Cat & Hammer & Bear-Trap \\
\hline Frog & Rabbit & Turtle & Pot & Faucet \\
\hline Frying-Pan & Pot & Spoon & Rabbit & Hamburger \\
\hline Garbage-Can & Glass & Saltshaker & Horse & Church \\
\hline Giraffe & Horse & Dinosaur & Basket & Saltshaker \\
\hline Glass & Bucket & Garbage-Can & Deer & Scissors \\
\hline Goat & Deer & Dog & Bucket & Ship \\
\hline
\end{tabular}


APPENDIX (Continued)

\begin{tabular}{|c|c|c|c|c|}
\hline \multirow{3}{*}{$\begin{array}{c}\text { Target } \\
\text { Picture } \\
\text { Presented } \\
\text { Gorilla }\end{array}$} & \multicolumn{4}{|c|}{ Written Distractors } \\
\hline & \multicolumn{2}{|c|}{ Visually Similar Items } & \multicolumn{2}{|c|}{ Visually Dissimilar Items } \\
\hline & Sloth & Toddler & Fly & Vase \\
\hline Grasshopper & Fly & Beetle & Sloth & T-Shirt \\
\hline Gun & Hammer & Electric-Mixer & Staircase & Shopping Cart \\
\hline Harp & Staircase & Pillar & Donkey & Electric-Mixer \\
\hline Hat & Boat & Hamburger & Windmill & Gerbil \\
\hline Helicopter & Windmill & Faucet & Boat & Cat \\
\hline Horse & Cow & Donkey & Barn & Bicycle \\
\hline House & Barn & Church & Cow & Scarf \\
\hline Iron & Telephone & Ship & Table & Cat \\
\hline Ironing-Board & Table & Scissors & Telephone & Rabbit \\
\hline Jacket & Sweater & T-Shirt & Hamster & Tree \\
\hline Kangaroo & Hamster & Gerbil & Sweater & Heron \\
\hline Kettle & Bucket & Basket & Wagon & Acorn \\
\hline Lamp & Mushroom & Vase & Tiger & Church \\
\hline Leopard & Tiger & Cat & Mushroom & Turkey \\
\hline Lion & Cat & Dog & House & Dolphin \\
\hline Monkey & Squirrel & Cat & Car & Couch \\
\hline Motorcycle & Car & Bicycle & Squirrel & Handbag \\
\hline Mouse & Otter & Rabbit & Lamp & Spoon \\
\hline Mushroom & Lamp & Tree & Flamingo & Hippopotamus \\
\hline Ostrich & Flamingo & Heron & Dog & Ladle \\
\hline Owl & Eagle & Acorn & Shorts & Rat \\
\hline Pants & Shorts & Scarf & Eagle & Squirrel \\
\hline Peacock & Porcupine & Turkey & Fish & Book \\
\hline Penguin & Fish & Dolphin & Binoculars & Elephant \\
\hline Piano & Vanity & Couch & Otter & Wheel-Chair \\
\hline Pig & Sheep & Hippopotamus & Vanity & Hang-Glider \\
\hline Pipe & Ladle & Spoon & Sheep & Ostrich \\
\hline Pitcher & Cup & Handbag & Skunk & Rocket \\
\hline Pot & Ladle & Frying-Pan & Groundhog & Beetle \\
\hline Rabbit & Groundhog & Rat & Frying-Pan & Rug \\
\hline Racoon & Skunk & Squirrel & Cup & Leaf \\
\hline Record-Player & Suitcase & Book & Boot & Hippopotamus \\
\hline Rhinoceros & Elephant & Hippopotamus & Suitcase & Sweater \\
\hline Rocking-Chair & Chair & Wheel-Chair & Skateboard & Cow \\
\hline Roller-Skate & Skateboard & Binoculars & Chair & Squirrel \\
\hline Rooster & Turkey & Ostrich & Windsurfer & Door \\
\hline Sailboat & Windsurfer & Hang-Glider & Turkey & Shell \\
\hline Saltshaker & Garbage-Can & Lighthouse & Arrow & Dragon \\
\hline Sea-Horse & Lizard & Dragon & Fridge & Mouse \\
\hline Seal & Fish & Dog & Crate & Scarecrow \\
\hline Sheep & Pig & Cow & Ear & Microwave \\
\hline Shirt & T-Shirt & Sweater & Lizard & Bicycle \\
\hline Shoe & Slipper & Boot & Racoon & Lighthouse \\
\hline Skunk & Racoon & Squirrel & Slipper & Table \\
\hline Sled & Gate & Door & Dog & Magnifying Glass \\
\hline Snail & Ear & Shell & Pig & Table \\
\hline Snowman & Clown & Scarecrow & Ant & Box \\
\hline Spider & Ant & Beetle & Clown & Zebra \\
\hline Spinning-Wheel & Cannon & Bicycle & Rabbit & Ant \\
\hline Squirrel & Rabbit & Mouse & Cannon & Jacket \\
\hline Stool & Chair & Table & T-Shirt & Bus \\
\hline Stove & Fridge & Microwave & Shirt & Ant \\
\hline Strawberry & Raspberry & Orange & Fly & Kettle \\
\hline Swan & Snake & Leaf & Couch & Alarm-Clock \\
\hline Sweater & Shirt & Jacket & Door & Butterfly \\
\hline Swing & Door & Box & Raspberry & Snail \\
\hline Telephone & Gas-Meter & Alarm-Clock & Lasso & Tiger \\
\hline Tie & Lasso & Magnifying Glass & Gas-Meter & Trailer \\
\hline
\end{tabular}


APPENDIX (Continued)

\begin{tabular}{lllll}
\hline \multicolumn{1}{c}{$\begin{array}{c}\text { Target } \\
\text { Picture } \\
\text { Presented }\end{array}$} & \multicolumn{4}{c}{ Written Distractors } \\
\cline { 2 - 5 } Tiger & Leopard & Zebra & Truck & Faucet \\
Train & Truck & Bus & Leopard & Window \\
Tree & Cloud & Cauliflower & Life-Jacket & Car \\
Truck & Train & Bus & Spider & Door-Knob \\
Turtle & Dinosaur & Snail & Helicopter & Vase \\
Vest & Life-Jacket & Butterfly & Cloud & Bat \\
Wagon & Trailer & Baby-Carriage & Bucket & Lizard \\
Watering-Can & Bucket & Kettle & Dinosaur & Sock \\
Well & House & Window & Leopard & Truck \\
Windmill & Helicopter & Faucet & Vase & Plate \\
Wineglass & Vase & Door-Knob & Motorcycle & Cow \\
Zebra & Leopard & Tiger & House & Cheese \\
\hline
\end{tabular}

(Manuscript received March 31, 1997;

revision accepted for publication June 2, 1997.) 